\title{
Molecular Dynamics Simulation of Crosslinked Epoxy Resins: Curing and Mechanical Properties
}

\author{
Tomonaga Okabe ${ }^{\mathrm{a}}$, Yutaka Oya ${ }^{\mathrm{a}, *}$, Koichi Tanabe ${ }^{\mathrm{a}}$, Gota Kikugawa ${ }^{\mathrm{b}}$, Kenichi \\ Yoshioka $^{\text {c }}$ \\ ${ }^{a}$ Department of Aerospace Engineering, Tohoku University, 6-6-01, Aoba-yama, Aoba-ku, \\ Sendai, Miyagi, 980-8579 Japan \\ ${ }^{b}$ Institute of Fluid Science, Tohoku University, 2-2-1, Katahira, Aoba-ku, Sendai, Miyagi, \\ 980-8577 Japan \\ ${ }^{c}$ Toray Composites (America), Inc., 19002 50th Avenue East, Tecoma, WA 98446, United \\ States Composite Materials Reserch Laboratories (CMRL), Toray Industries, Inc.
}

\begin{abstract}
We performed molecular dynamics (MD) simulation that includes multidisciplinary characteristics from synthesis to mechanical properties of epoxy resin. First, to reproduce the actual chemical reaction between matrix and curing agents, we conducted curing simulation wherein the activation energy and heat of formation are considered for the chemical reaction. Subsequently, we performed MD simulations using cross-linked structure obtained from curing simulation to derive density and Young's modulus. Results indicated that crosslinked structures involving both activation energy and heat of formation could reproduce experiment results that are evaluated using differential scanning calorimetry (DSC) measurements and mechanical tests. The simulated results imply that electrostatic interaction plays an important role in Young's modulus. The density of the hydrogen bond between the oxygen of the hydroxyl group and the hydrogen atom is a key factor for the difference in Young's modulus for each base resin. These findings confirm that MD simulation is a potential alternative to experiments for the appropriate material selection of epoxy resin.
\end{abstract}

Keywords: Curing characteristics, Thermosetting resin, Molecular dynamics, Mechanical properties

\section{Introduction}

Polymer matrix composites (PMCs) are key structural materials in transportation systems. Because of their lighter weight and grater stiffness, compared to conventional materials such as aluminium, half of the conventional metal in

\footnotetext{
* Corresponding author

Email address: oya@plum.mech.tohoku.ac.jp (Yutaka Oya)
}

Preprint submitted to European Polymer Journal

February 17, 2016

(C) 2016. This manuscript version is made available under the Elsevier user license http://www.elsevier.com/open-access/userlicense/1.0/ 
the airframe of a Boeing 787 has been replaced by carbon-fibre-reinforced plastics (CFRPs) to reduce the aircraft's overall weight and extend its cruising range [1].

Generally, thermosetting resin is utilized for the matrix in CFRPs. Epoxy resin is widely used, because of its high thermostability and/or strong adhesion to carbon fibres [2]. Development of the properties of epoxy resin may dramatically improve the mechanical performance of composite materials. For instance, Kim et al. demonstrated that the fracture toughness of CFRP is clearly improved by selecting appropriate resin [3]. Since there are numerous types of epoxy resin, the experimental selection of matrix resin of PMC is expensive and time-consuming. For this reason, molecular dynamics (MD) simulation is expected to play an important role in the appropriate design of matrix resin (e.g., screening target materials among numerous candidates and proposing new combinations of base resin and curing agent in accordance with certain situations.) In fact, Jaramillo et al. conducted MD simulations and described the crosslinked structures of epoxy resin for precise optimization of aircraft design that considers a wide range of material behaviours, from molecular to macro-scale [4].

Many researchers have attempted to predict glass-transition temperature $\left(T_{\mathrm{g}}\right)$ and mechanical properties of thermosetting resin [5-11]. Fan et al. accurately predicted the $T_{\mathrm{g}}$ of an epoxy resin using MD simulation [12]. Komarov et al. performed coarse-grained MD simulations and demonstrated that $T_{\mathrm{g}}$ obviously increases with increased crosslinked density of resin [13]. Izumi et al. also found this dependence between the crosslinked density and $T_{\mathrm{g}}$ to be true for phenol resin [14]. Additionally, Soni et al. indicated that $T_{\mathrm{g}}$ decreases with increased degree of polymerization of a curing agent [15]. Li et al. used MD simulation for extensive characterization of the thermo-mechanical response of a thermoset polymer, and evaluated Young's modulus and $T_{\mathrm{g}}$ [16-18]. Dong et al. found that high moisture content and high temperatures weaken the mechanical properties of epoxy resin [19]. Li et al. and Jeyranpour et al. indicated that curing agents greatly influence glass transition and mechanical properties $[20,21]$. Moreover, Yang et al. predicted the failure of epoxy resin by coarsegrained models [22]. They found that yielding in the compounds is caused by shear under low hydrostatic tension and by cavitation under high hydrostatic tension [23].

In addition to these properties, chemical properties (e.g., curing characteristics) are important for selecting the matrix resin of PMC, since they are directly linked to the molding process, which greatly influences both the mechanical properties and productivity of industrial products. Nevertheless, curing simulation has not been studied at satisfactory level until recently, since only steric repulsion between molecules has been considered a criterion of curing reaction. To simulate the detailed mechanism of curing reaction, we recently proposed a new curing simulation technique based on MD simulation, taking into account the activation energy and heat of formation for the chemical reaction using molecular orbital (MO) calculation [24]. This approach can reproduce curing characteristics, depending on combinations of base resins and curing agents and has been implemented in Materials Studio software [25]. However, properties 
other than curing characteristics were not involved in the previous study, and we have not investigated whether simulated cross-linked structures can reproduce properties other than curing characteristics. Naturally, from the practical viewpoint, simulation that covers both synthesis and physical properties (including density and Young's modulus) of the thermosetting resin is desired for a method that could serve as an alternative to the experiment.

In this study, we conducted MD simulation to comprehensively evaluate the curing and mechanical properties of epoxy resin. Section 2 discuss our MD simulation of chemical properties for the curing process with the help of MO calculation. The simulated results were compared with the results of experiments obtained from differential scanning calorimetry (DSC) measurements. Section 3 describes our MD simulation of mechanical properties using the crosslinked structures obtained in the simulation discussed in section 2. Section 4 presents summary and conclusions.

\section{Curing simulation}

In this study on the chemical reaction between base resin and curing agents, we investigated four species that comprise combinations of base resin and curing agents (Table 1 and Fig. 1). Figure 2 depicts a schematic of the chemical reaction of this simulation in the case of TGDDM/44DDS as an example. When the epoxy group of the base resin approaches the amino group of the curing agent, cross-links between epoxy and amino groups are constructed after passing a transition state. Figure 3 presents the potential energy curve versus the reaction pass in the system of TGDDM/44DDS. A potential barrier exists between the state before the curing reaction and that after the chemical reaction. The state that corresponds to this highest potential energy is known as a transition state, and this barrier is known as activation energy. To reproduce the curing process, activation energy involved in a chemical reaction should be considered. Thus, we proposed a new MD simulation with the help of MO calculation [24, 26]. We calculated activation energy $(\Delta G)$ and heat of formation $\left(\Delta H_{\mathrm{f}}\right)$ by a semi-empirical MO method using the PM6 Hamiltonian with the MOPAC2009 program, and these elements are built in the MD method. Two successive reactions for TGDDM/44DDS are denoted as "first" in Fig. 4(a) and "second" in Fig. 4(b).

Table 1: Mixing ratio for each resin-epoxide combination.

\begin{tabular}{cccc}
\hline & Target materials & Mixing ratio & Number of atoms \\
\hline$\# 1$ & DGEBA/DEGBA8/33DDS & $16 / 1 / 9$ & 4225 \\
$\# 2$ & DGEBA/DEGBA8/44DDS & $16 / 1 / 9$ & 4225 \\
$\# 3$ & TGDDM/33DDS & $1 / 1$ & 4860 \\
$\# 4$ & TGDDM/44DDS & $1 / 1$ & 4860 \\
\hline
\end{tabular}




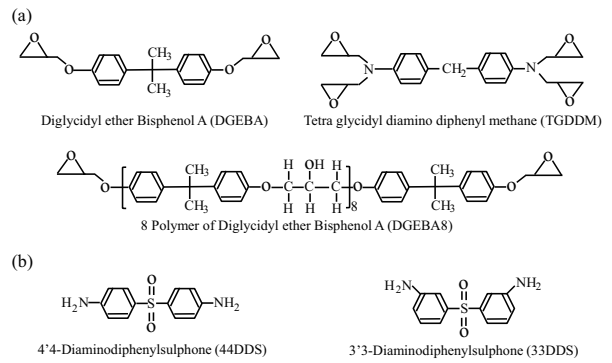

Figure 1: Molecular structures of (a) base resins and (b) curing agents that constitute target materials (\#1-\#4) in Table 1. Both DGEBA and DGEBA8 that are mixed in \#1 and \#2 have two epoxy groups. On the other hand, TGDDM that is used in \#3 and \#4 has four epoxy groups.

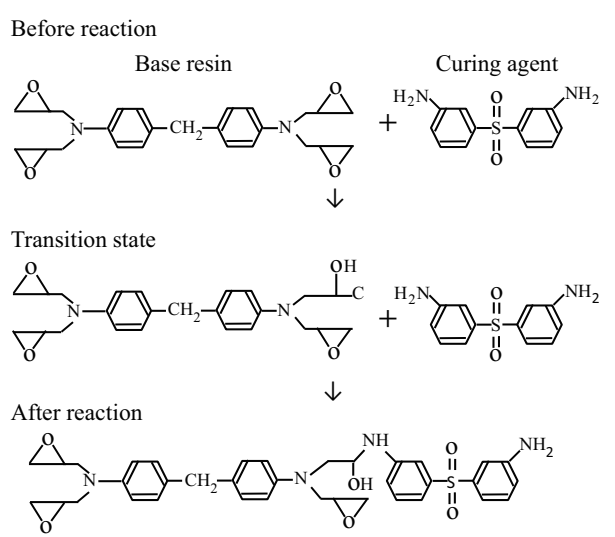

Figure 2: Schematic for the chemical reaction (TGDDM/44DDS) during cross-linking.

Before curing calculation, we must obtain an initial condition in which the free energy of the system is minimized by relaxation calculation. The relaxation procedure consists of the following two steps.

(1) The monomers of the base resin and the curing agent are randomly packed into a simulation cell under-three dimensional (3D) periodic boundary conditions (Fig. 5).

(2) MD calculation is performed for 2ns under the NPT ensemble, where pressure is constrained by atmospheric pressure and temperature is constrained by room temperature.

After relaxation of the system, curing calculation was carried out under the NPT ensemble to reproduce the cross-linked structure of epoxy resin. When the epoxy group and the amino group approached one another within the reaction range $(0.6 \mathrm{~nm})$, they are wrapped into one functional group. Hereafter, a random 


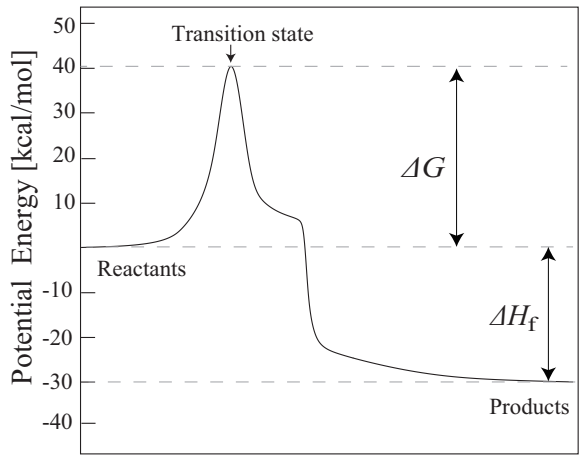

Reaction Pass

Figure 3: Curing curve obtained using MO calculation. Potential energy is plotted against reaction pass. Activation energy $(\Delta G)$ is determined by the difference in potential energies between the transition state and before-reaction state. The heat of formation $\left(\Delta H_{\mathrm{f}}\right)$ is the difference between before- and after- reaction states.

number $P(0$ to 1$)$ is assigned to the functional group and compared with the reaction probability $k$, defined as

$$
k=\mathrm{A} \exp \left(-\frac{\Delta G}{\mathrm{RT}}\right)
$$

where $\mathrm{A}$ is the acceleration factor $\left(1 \times 10^{8}\right), \Delta G$ is the activation energy determined using the MO method, $\mathrm{R}$ is the gas constant, and $T$ is the local temperature. If $P$ is less than the reaction probability, curing reaction takes place and the cross-linked structure is obtained. This reaction causes conversions of bonding modes among atoms. Therefore, the force field parameters are reassigned to be consistent with those of the cross-linked structure. Finally, the heat of formation, which arises from the curing reaction, is added as the increase of kinetic energy of the product. The heat of formation after the reaction can be written as

$$
\Delta H_{\mathrm{f}}=K_{\text {after }}-K_{\text {before }}
$$

where $\Delta H_{\mathrm{f}}$ is the heat of formation, $K_{\text {after }}$ is the kinetic energy after the reaction, and $K_{\text {before }}$ is the kinetic energy before the reaction. This heat of formation raises the temperature at least locally. Since the reaction barrier is effectively formed by $\Delta G / \mathrm{R} T$, as temperature increases, a curing rate increases. Therefore, curing is a positive-feedback reaction. While local temperature changes depending on local position, the system temperature is controlled by a heat bath. In our simulation, the system temperature is initially set to $27^{\circ} \mathrm{C}$ and rises $0.1^{\circ} \mathrm{C} / \mathrm{ps}$ until the system temperature reaches $1000^{\circ} \mathrm{C}$. The AMBER force field was assigned as the interaction force between molecules [27].

To confirm our simulation method, the differences in curing reactivity are experimentally compared with those using Differential Scanning Calorimetry 


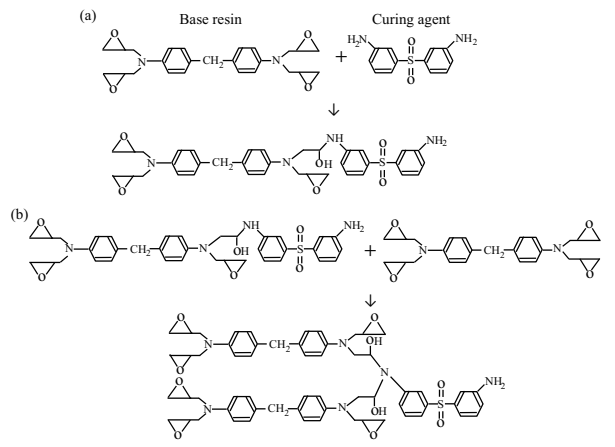

Figure 4: Structural formulae (TGDDM/44DDS) for the (a) first and (b) second curing reactions.

Table 2: Activation energy $(\Delta G)$ and heat of formation $\left(\Delta H_{\mathrm{f}}\right)$ in each curing reaction for resinepoxide combinations in Table 1 . Those for the first and second curing reactions depicted in Fig. 3 are also indicated.

\begin{tabular}{cccc}
\hline & Combination & $\Delta G(\mathrm{kcal} / \mathrm{mol})$ & $\Delta H_{\mathrm{f}}(\mathrm{kcal} / \mathrm{mol})$ \\
\hline$\# 1$ & 33DDS-DGEBA 1st/2nd & $37.59 / 39.96$ & $32.91 / 30.06$ \\
$\# 1$ & 33DDS-DGEBA8 1st/2nd & $39.20 / 39.20$ & $31.06 / 31.06$ \\
$\# 2$ & 44DDS-DGEBA 1st/2nd & $42.01 / 40.79$ & $28.31 / 22.05$ \\
$\# 2$ & 44DDS-DGEBA8 1st/2nd & $40.90 / 40.90$ & $30.27 / 30.27$ \\
$\# 3$ & 33DDS-TGDDM 1st/2nd & $41.92 / 39.66$ & $26.03 / 30.15$ \\
$\# 4$ & 44DDS-TGDDM 1st/2nd & $43.68 / 43.81$ & $26.07 / 28.87$ \\
\hline
\end{tabular}

(DSC) (DSCQ200; TA Instruments). In our simulation, degree of cross-linking $D$ is defined as:

$$
D=\frac{n_{\text {react }}}{n_{\text {total }}}
$$

where $n_{\text {react }}$ is the number of epoxy groups that reacted with the amino group, and $n_{\text {total }}$ is the total number of epoxy groups before the curing reaction. In contrast, curing conversion $C$ in experiments is defined by $[28,29]$ :

$$
C=\frac{1}{\Delta H} \int \phi d t
$$

where $\Delta H$ is the total enthalpy of the chemical reaction and $\phi$ is heat flow. The initial temperature is set to $50^{\circ} \mathrm{C}$ for all combinations in Table 1 . System temperatures are increased $3{ }^{\circ} \mathrm{C} / \mathrm{min}$ for $\# 1$ and $\# 2$, and $10^{\circ} \mathrm{C} / \mathrm{min}$ for $\# 3$ and \#4 until the temperature reaches $300^{\circ} \mathrm{C}$.

Curing characteristics were investigated and compared with experiment data. Figure 6 depicts both the experimental and simulated curing curves of four resins. Both curing curves have the same characteristics in these profiles. First, curing starts at a finite temperature because a certain temperature is needed 


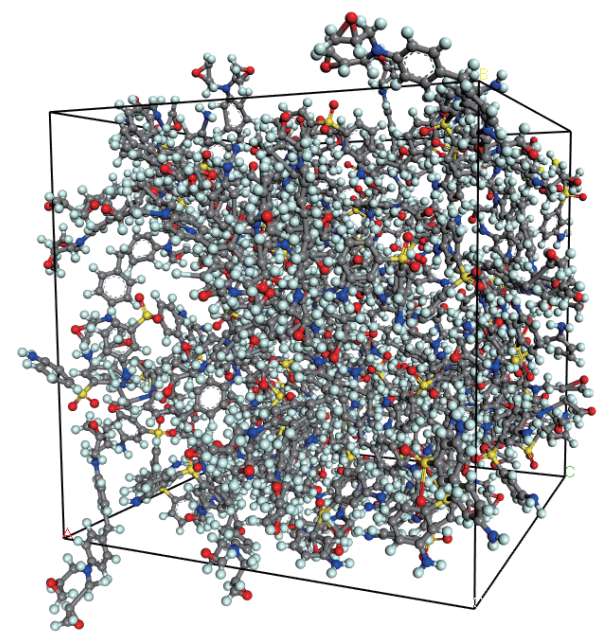

Figure 5: Initial structure before curing reaction. In this system, the matrix resins and curing agents are randomly packed in a $3 \mathrm{D}$ periodic cell.

to overcome activation energy. Once the curing reaction begins, the reaction is accelerated by the generated heat of formation. Therefore, slopes of curing curves gradually increase at the beginning of the curing reaction. Finally, the slopes gradually decrease due to the reduction of contact probability between base resin and curing agent. Consequently, the curing curve in both experiments and our simulation results is hyperbolic-tangent.

Although many characteristics of the profile in the curing curve can be reproduced in our simulation, some differ from those in the experiment. In our simulation, the curing reaction occurred throughout a wider temperature range. We assume that this difference is due to the difference in heating rate between our simulation $\left(1.0 \times 10^{11^{\circ}} \mathrm{Cs}^{-1}\right)$ and the experiment $\left(3.0-10.0^{\circ} \mathrm{Cmin}^{-1}\right)$. Moreover, we did not obtain $D=100 \%$, even though the temperature reached 1000 ${ }^{\circ} \mathrm{C}$ in the simulation due to the difference in the definitions of $C$ and $D$. In our simulation, $D$ is defined as the ratio of the number of epoxy groups after curing reaction and before curing reaction. However, $C$ is obtained from enthalpy and heat flow Eq. (4). From this equation, $C$ is normalized by the total enthalpy of curing reaction. Therefore, when the reaction is finished in experiments, $D$ is regarded as $100 \%$ even if reaction groups remain in the system.

Figure 6 plot the dependences of $C$ and $D$ on two curing agents, 33DDS and 44DDS. In both experiments and simulations, the curing reactions with 33DDS are higher than those with 44DDS. This difference is due to the difference in activation energy and heat of formations: curing reaction with 33DDS has lower activation energy and higher heat of formation, as indicated in Table 2. The difference in activation energy between 33DDS and 44DDS is attributed to the difference in stability. Namely, 33DDS is so unstable that it is highly 
(a)

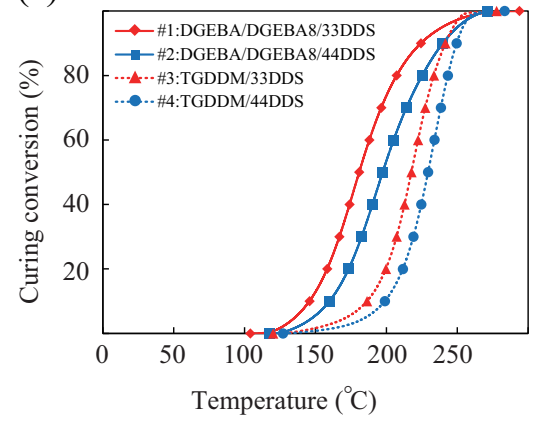

(b)

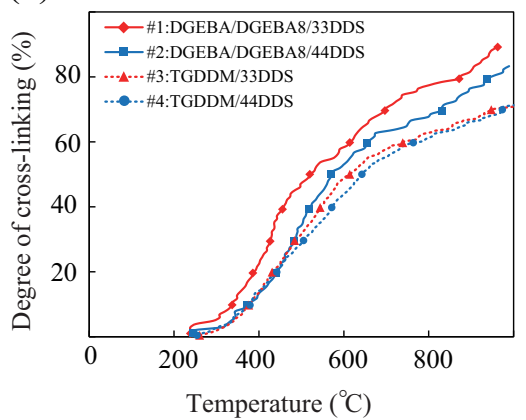

Figure 6: Temperature dependence of curing curves obtained by (a) DSC experiments and (b) simulations. These curves represent the epoxy-resin combinations in Table $1(\# 1(\mathbf{O}), \# 2(\boldsymbol{\square})$ $\# 3(\mathbf{\Delta})$, and $\# 4(\boldsymbol{\bullet}))$.

reactive (i.e., lower activation energy). In fact, MO calculation confirms that internal energy of 33DDS is $10 \%$ higher than that of 44DDS. As depicted in Fig. 1 (b), the difference between 33DDS and 44DDS structures is the location of the amine groups. The amine group $\left(-\mathrm{NH}_{2}\right)$ and sulfonyl group $\left(-\mathrm{S}(=0)_{2^{-}}\right)$of 33DDS are in the meta-position of benzene rings, and those of 44DDS are in the para-position. Thus, by resonance form of benzene rings, 44DDS becomes more stable than 33DDS. Finally, in our simulation, when temperature increased, curing reactions in the materials achieved $D=60 \%$, in the order of $\# 1, \# 2, \# 3$, and \#4. Likewise, the order of $C$ in the experiments was \#1, \#2, \#3, and \#4. In the previous study, we could simulate the curing process for six combinations of material systems. Moreover, a knee point found in the experimental curing curve for the case of DGEBA/DETA/44DDS, which mixed the curing agents DETA/44DDS, could be successfully reproduced considering the activation energy and heat of formation. It is worth noting that the curing characteristics for ten combinations of materials could be successfully represented by curing simulations in present and previous studies [24]. This agreement of curing tendency between the simulation and the experiment is assumed to originate from the magnitude relationship between activation energy and heat of formations (Table 2); for example, the material that needs high activation energy and generates low heat of formation in the curing reaction has low reactivity. Therefore, activation energy and heat of formation are essential for describing curing reactions, and the chemical curing simulation that is presented in this study has the potential to sufficiently reproduce the experiment results. 
Table 3: Densities of curing products with $T=298 \mathrm{~K}$ for the present simulation and the synthetic experiment for each epoxy-resin combination.

\begin{tabular}{cccc}
\hline & & $\begin{array}{c}\text { Simulation } \\
\left(\mathrm{g} / \mathrm{cm}^{3}\right)\end{array}$ & $\begin{array}{c}\text { Experiment } \\
\left(\mathrm{g} / \mathrm{cm}^{3}\right)\end{array}$ \\
\hline$\# 1$ & DGEBA/DGEBA8/33DDS & 1.238 & 1.223 \\
$\# 2$ & DGEBA/DGEBA8/44DDS & 1.228 & 1.219 \\
$\# 3$ & TGDDM/33DDS & 1.289 & 1.290 \\
$\# 4$ & TGDDM/44DDS & 1.293 & 1.290 \\
\hline
\end{tabular}

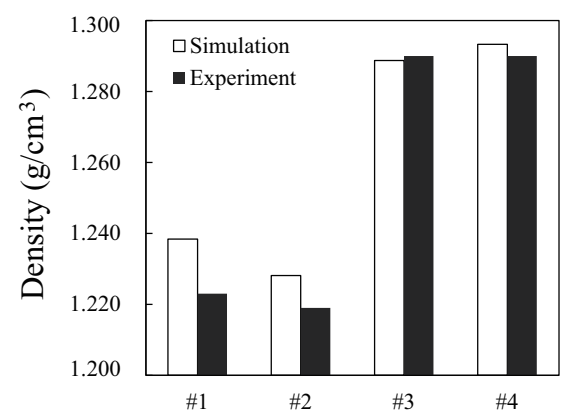

Figure 7: Magnitude relationship of densities among the curing products for the present simulations $(\square)$ and the synthetic experiments (ם) for each epoxy-resin combination.

\section{MD simulation with cross-linked structures and estimation of me- chanical properties}

In the previous section, we simulated the curing characteristics of the materials listed in Table 1. In this section, we evaluated density and Young's modulus for systems with $60 \%$ curing conversion in these materials obtained in the previous curing simulation. We applied the TEAM force field [30] to the potential field and utilized LAMMPS software [31] to perform MD simulation. Prior to conducting MD simulation, we translated the structure data obtained by curing simulation into LAMMPS format and assigned Force-Field parameters using Direct Force Field 7.1 standard edition [30]. Here, it should be noted that only the van der Waals radius in the force field parameters was slightly adjusted so as to reproduce those densities in the experiments. In addition to the van der Waals parameters, various force-field parameters are involved in constructing a molecular structure (e.g., bonding potential between neighbor atoms, angular potential, and long-range coulomb potential). These parameters are optimized to reproduce thermodynamic properties and structures of low weight molecular liquids. Thus, polymer systems are not mimicked as correctly as molecular liquids using conventional force-field parameters. Therefore, the parameters must be adjusted so as to reproduce experiment results. As a 
Table 4: Young's modulus of curing products with $T=298 \mathrm{~K}$ for the present simulation and the tensile experiment for each epoxy-resin combination. In the simulation results, Young's moduli both with and without coulomb potential (c.p.) are described.

\begin{tabular}{ccccc}
\hline & & $\begin{array}{c}\text { Simulation:w/(c.p.) } \\
(\mathrm{GPa})\end{array}$ & $\begin{array}{c}\text { Simulation:w/o(c.p.) } \\
(\mathrm{GPa})\end{array}$ & $\begin{array}{c}\text { Experiment } \\
(\mathrm{GPa})\end{array}$ \\
\hline$\# 1$ & DGEBA/DGEBA8/33DDS & 3.11 & 1.96 & 3.42 \\
$\# 2$ & DGEBA/DGEBA8/44DDS & 3.09 & 1.99 & 3.21 \\
$\# 3$ & TGDDM/33DDS & 4.46 & 2.58 & 4.37 \\
$\# 4$ & TGDDM/44DDS & 4.06 & 2.32 & 4.02 \\
\hline
\end{tabular}

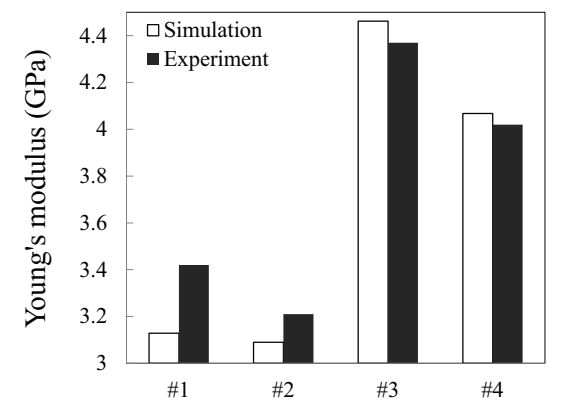

Figure 8: Magnitude relationship of Young's moduli among the curing products for the present simulations $(\square)$ and the tensile experiments ( $)$ for each epoxy-resin combination.

result of the adjustment, we found that every atomic distance parameter in the van der Waals potential should be 0.927 times those used by the TEAM force field. Afterwards, the structures were relaxed according to the following procedure at constant temperature $(T=298 \mathrm{~K})$. First, the structures obtained by curing simulation were packed into a simulation cell under 3D periodic boundary conditions. Second, relaxation was performed in the NPT ensemble for $5 \mathrm{~ns}$. Densities were obtained from the averaged cell volume during $5 \mathrm{~ns}$ in the relaxation. Subsequently, uniaxial tensile simulations were performed with a strain rate of $5 \times 10^{8} \mathrm{~s}^{-1}$ until longitudinal strain reached $15 \%$. To suppress the typical fluctuation of stress in MD simulation, the stress-strain curves were averaged using three different production runs. Young's modulus was obtained by linear fitting the averaged-stress-strain curves up to $3 \%$ tensile strain.

In Table 3, simulated densities are compared with the experiment results obtained using hydrostatic weighting. The simulation results reproduce the experiment data quite well by adjusting the van der Waals radius. Figure 7 indicates the magnitude relationship of density between simulation and experiment results. Table 4 and Fig. 8 compare Young's modulus in the experiment 

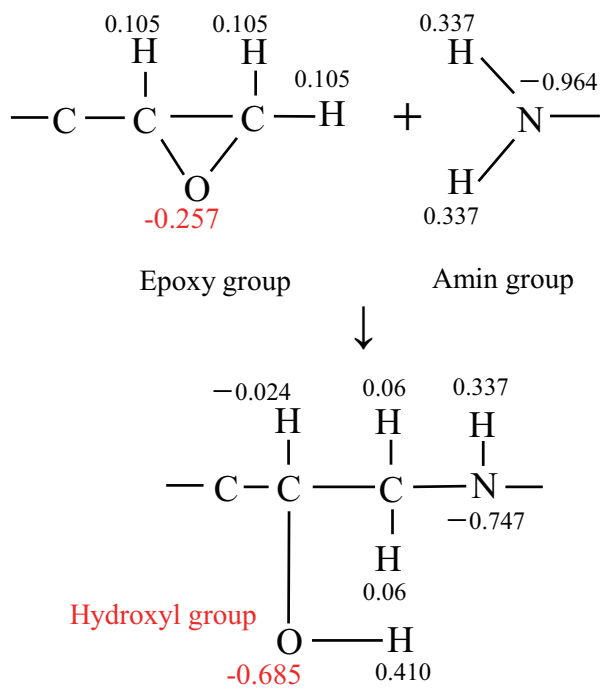

Figure 9: Distributions of electrical static charge of functional groups before and after the chemical reaction. The oxygen atom acquires more negative charge by forming the hydroxyl group generated by the chemical reaction between the epoxy group and the amine group.

with those in our simulation. The differences are less than 10\%. Taking into consideration the difference in strain rates between the experiment and our simulation, we conclude that our results agree well with the experiment results.

Tensile simulations without the coulomb potential are also simulated to investigate the physical origin of differences in Young's modulus among four combinations (\#1 through \#4 in Table 1). Results indicate that Young's moduli with the coulomb potential are about $60 \%$ larger on average than those without it. Therefore, we concluded that the coulomb potential definitely influences differences in Young's modulus.

Hereafter, changes in Young's modulus (Fig.8) are discussed from the viewpoint of the coulomb potential. Although our system does not contain ionic molecules, each molecule is polarized, and the electrostatic interaction among molecules plays an important role in the system stiffness. An attractive force due to electrostatic interactions generates cohesive force between molecules and leads to an increment of Young's modulus.

Figure 9 depicts changes in charge distributions of epoxy resin before and after curing. The epoxy group changes to a hydroxyl group, and the oxygen atom in these groups becomes more negatively charged throughout the reaction. The oxygen atom in the hydroxyl group strongly attracts the hydrogen atom in other molecules, because of a hydrogen bond. The radial distribution function of oxygen in the epoxy group (solid line) and that in the hydroxyl group (dashed line) for all atoms are presented in Fig. 10. The strong peaks found in the dashed line indicate that atoms exist adjacent to the oxygen atom 


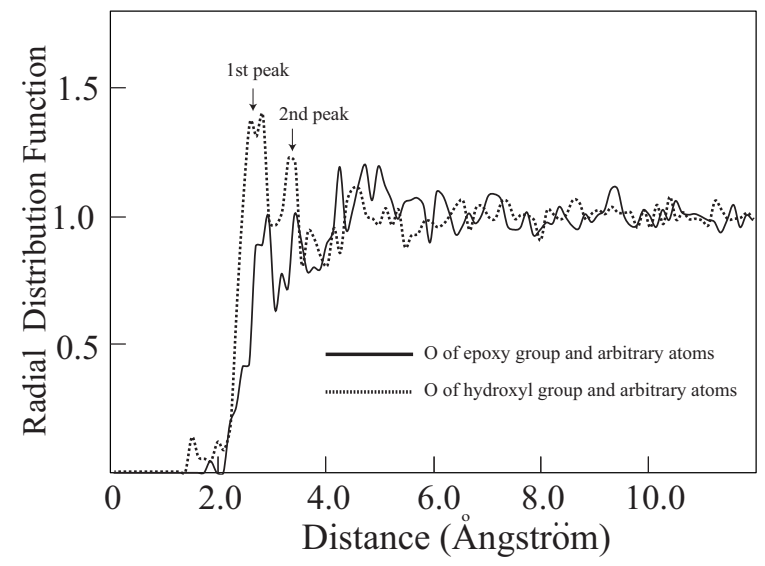

Figure 10: Radial distribution function between the oxygen atom in the epoxy group and other arbitrary atoms (solid line), and that between the oxygen atom in the hydroxyl group and other arbitrary atoms (dashed line). In contrast with the solid line, the dashed line has two different peaks (1st and 2nd peaks) at a distance of $3 \AA$.

of the hydroxyl group. To identify the types of atoms, Fig. 11 plots two radial distribution functions in the oxygen atom of the hydroxyl group: the solid line denotes hydrogen atoms, and the dashed line, which has been also drawn in Fig. 10, denotes all atoms. The radial distribution for the hydrogen atoms has two different peaks, like that for all atoms (solid line); these two peaks overlap. Considering the distance between hydrogen and oxygen atoms in the same hydroxyl group, these two peaks do not represent the distance in the hydroxyl group (we confirmed that the peak at $1.5 \AA$ originates from a hydroxyl group). Both the peaks imply hydrogen bond interaction between the hydrogen atom and the oxygen atom of the hydroxyl group. Figure 12(a) schematically depicts this hydrogen bond interaction. This hydrogen bond interaction creates network structures (Fig. 12(b)) and influences stiffness in the tensile experiment. Therefore, the number density of the hydroxyl group that causes hydrogen bond interaction is positively correlated with Young's modulus. Here, we introduce the number density $\rho\left(\AA^{-3}\right)$ of the hydroxyl group for each epoxy resin combination. In the present study, both \#1 and \#2 have almost same number density $(\rho=0.21)$. Likewise, $\rho=0.28$ for both \#3 and \#4. This result is attributed simply to the number of epoxy groups per monomer, since both curing agents (33DDS and 44DDS) have the same number of amine groups. Therefore, the curing products generated by TGDDM (\#3 and \#4) have a greater Young's modulus than those synthesized by DGEBA (\#1 and \#2), due to the number density of the hydroxyl group.

In contrast, the influence of the curing agents on Young's modulus is attributed to the dipole-dipole interaction of the cured epoxy resin. Table 5 lists 


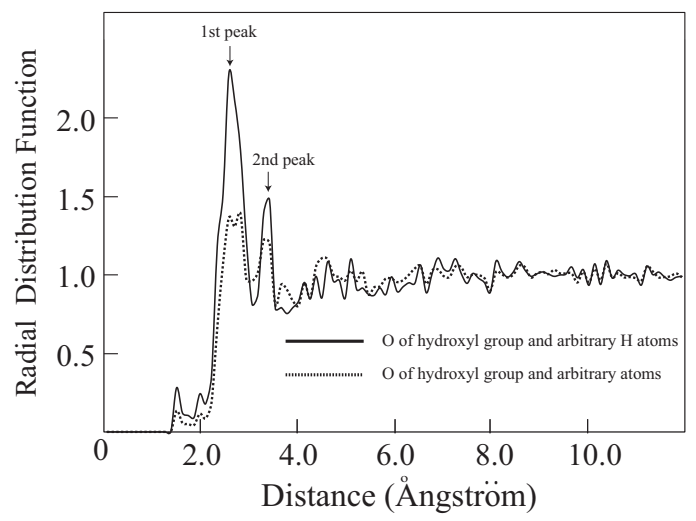

Figure 11: Radial distribution function between the hydrogen atom in the hydroxyl group and the arbitrary hydrogen atom (solid line), and that between the oxygen atom in the hydroxyl group and other arbitrary atoms (dashed line). Similar to the RDF profile denoted by the dashed line in Fig. 10, the solid line has two different peaks, and these peak positions coincide well with those of the dashed line in this figure.

(a)

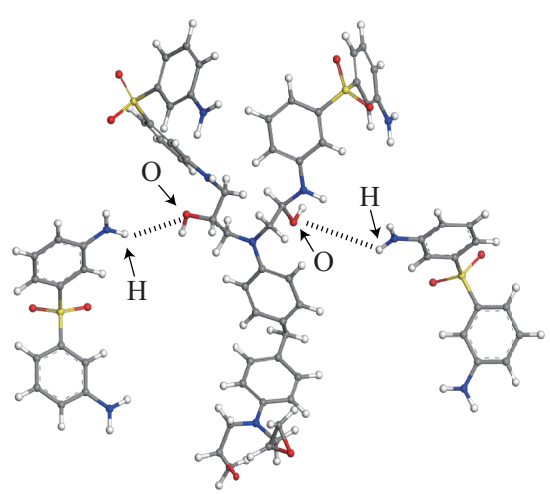

(b)

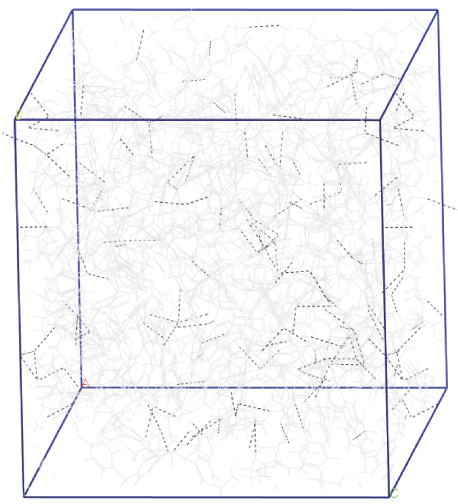

Figure 12: (a) Schematic figure of hydro-bonding interaction. Oxygen in the hydroxyl group interacts with the hydrogen atom by hydrogen bond interaction, leading to the two peaks of radial distribution functions in Figs. 10 and 11. (b) Snapshot of the hydrogen bond interactions that cause the network structures in the system. The molecular structures in this figure are displayed in light grey. In these figures, the hydro-bonding interaction is denoted by a dashed line.

the dipole moments of the curing products. The cross-linked resin with 33DDS has higher dipole moments than that with 44DDS, leading to the magnitude relation of Young's modulus (i.e., \#1 and \#3 are higher than \#2 and \#4). In 
Table 5: Dipole moments (debye) of curing products for the first and second reactions for each epoxy-resin combinations. The higher value of the two reactions in each combination is underlined.

\begin{tabular}{cccc}
\hline & Combination & $1^{\text {st }}$-reaction & $2^{\text {nd }}$-reaction \\
\hline$\# 1$ & DGEBA/33DDS & $\underline{9.516}$ & 6.586 \\
$\# 2$ & DGEBA/44DDS & 4.462 & $\underline{8.684}$ \\
$\# 3$ & TGDDM/33DDS & 8.472 & $\underline{\underline{10.439}}$ \\
$\# 4$ & TGDDM/44DDS & 8.164 & $\underline{8.801}$ \\
\hline
\end{tabular}

conclusion, the magnitude relationship of Young's modulus is in the order of \#3, \#4, \#1 and \#2 (Fig. 8). In this study, we investigated density and Young's modulus of thermosetting resin in full-atomistic MD simulation. Strength is also important for application to the transport industry; however, it is difficult to evaluate strength using MD simulation density. For example, strength is sensitive to microcracks and defects. Considerable computational resources are necessary to simulate these macroscopic phenomena using the full-atomic MD method, due to differences in space scales. Thus, reproduction of strength remains our future task.

\section{Conclusion}

We simulated the curing reaction of base resins (DGEBA, DGEBA8, and TGDDM) with two curing agents (33DDS and 44DDS) using MD simulation that considered both activation energy and heat of formation. Our simulations reproduced the features of curing curves in the experiments. Moreover, the curing reactions in our simulation proceeded in the order of DGEBA/DGEBA8/ 33DDS, DGEBA/DGEBA8/44DDS, TGDDM/33DDS, and TGDDM/44DDS. This difference in reactivity was also confirmed in our experiments. Young's modulus for the cured resin system obtained using our presented technique also agreed with experiment results after adjusting the van der Waals radius so as to reproduce the densities in the experiments. The influence of potential energy for Young's modulus for each resin was numerically examined. The simulated results confirmed that Young's modulus is strongly influenced by the coulomb potential. The density of the hydrogen bond between the oxygen of the hydroxyl group and the hydrogen atom generates the difference in Young's modulus for each base resin. The difference in dipole moment generates the difference in Young's modulus for each curing agent.

\section{Acknowledgements}

We acknowledge the support of the Cabinet Office, Government of Japan under a Cross-ministerial Strategic Innovation Promotion (SIP) Program. We also acknowledges the support of Toray Composites (America). 


\section{References}

[1] http://www.boeing.com/commercial/aeromagazine/airticles/qtr_4_06/airticle_04_2.html

[2] D. A. Papargyris, R. J. Day, A. Nesbitt, D. Bakavos., Comparison of the mechanical and physical properties of a carbon fibre epoxy composite manufactured by resin transfer moulding using conventional and microwave heating, Comp. Sci. \& Tech. 68 (2008) 1854-1861.

[3] J. K. Kim, C. Baillie, J. Poh, Y. W. Mai., Fracture toughness of CFRP with modified epoxy resin matrices, Comp. Sci. \& Tech. 43 (1992) 283-297.

[4] E. Jaramillo, N. Wilson, S. Christensen, J. Gosse, A. Strachan., Energybased criterion for PMMA from large-scale molecular dynamics simulations, Phys. Rev. B 85 (2012) 024114.

[5] L. J. Abbott, C. M. Colina, Atomistic structure generations and gas adsorption simulations of microporous polymer networks, Macromolecules 44 (2011) 4511-4519.

[6] N. Nouri, S. Z. Rad, A molecular dynamics investigation on mechanical properties of cross-linked polymer networks, Macromolecules 44 (2011) 5481-5489.

[7] L. J. Abbott, K. E. Hart, C. M. Colina, Polymeric: a generalized simulated polymerization algorithm for amorphous polymers, Theor. Chem. Acc. 132 (2013) 1334-1352.

[8] V. Varshney, S. S. Patnaik, A. K. Roy, B. L. Farmer, A molecular dynamics study of epoxy-based networks: cross-linking procedure and prediction of molecular material properties, Macromolecules 41 (2008) 6837-6842.

[9] C. Li, E. Coons, A. Strachan, Material property prediction of thermoset polymers by molecular dynamics simulations, Acta. Mech. 225 (2014) 11871196.

[10] S. Yang, J. Qu, Computing thermomechanical properties of crosslinked epoxy by molecular dynamics simulations, Polymer 53 (2012) 4806-4817.

[11] A. Shokuhfar, B. Arab, The effect of cross linking density on the mechanical properties and structure of the epoxy polymers: molecular dynamics simulation, J. Mol. Model. 19 (2013) 3719-3731.

[12] H. B. Fan, M. M. F. Yuen., Material properties of the cross-linked epoxy resin compound predicted by molecular dynamics simulation, Polymer 48 (2007) 2174-2178.

[13] P. V. Komarov, C. Y. Tsung, C. S. Ming, P. G. Khalatur, P. Reineker., Highly cross-linked epoxy resins: an atomic molecular dynamics simulation combined with a mapping/reverse mapping procedure, Macromolecules 40 (2007) 8104-8113. 
[14] A. Izumi, T. Nakao, M. Shibayama., Atomistic molecular dynamics study of cross-linked phenolic resins, Soft Matter 8 (2012) 5283-5292.

[15] N. J. Soni, P. H. Lin, R. Khare., Effect of cross-linker length on the thermal and volumetric properties of cross-linked epoxy networks: a molecular simulation study, Polymer 53 (2012) 1015-1019.

[16] C. Li, A. Strachan., Molecular simulations of crosslinking process of thermosetting polymers, Polymer 51 (2010) 6058-6070.

[17] C. Li, A. Strachan., Molecular dynamics predictions of thermal and mechanical properties of thermoset polymer EPON862/DETDA, Polymer 52 (2011) 2920-2928.

[20] C. Li, G. A. Medvedev, E. W. Lee, J. Kim, J. M. Caruthers, A. Strachan., Molecular dynamics simulations and experimental studies of the thermomechanical response of an epoxy thermoset polymer, Polymer 53 (2012) 4222-4230.

[19] D. R. Xin, Q. Han, Molecular dynamics study on the tensile deformation of cross-linking epoxy resin, J. Mol. Model. 53 (2015) 21-5.

[20] C. Li, E. Coons, A. Strachan, Material property prediction of thermoset polymers by molecular dynamics simulations, Act. Mech. 225 (2014) 11871196.

[21] F. Jeyranpour, Gh. Alahyarizadeh, B. Arab, Comparative investigation of thermal and mechanical properties of cross-linked epoxy polymers with different curing agents by molecular dynamics simulation, J. Mol. Graph. Mod. 62 (2015) 157-164.

[22] S. Yang, Z. Cui, J. Qu, A coarse-grained model for epoxymolding compound, J. Chem. Phys. 118 (2014) 1660-1669.

[23] S. Yang, J. Qu, Coarse-grained molecular dynamics simulations of the tensile behavior of a thermosetting polymer, Phys. Rev. E 90 (2014) 120601.

[24] T. Okabe, T. Takehara, K. Inose, N. Hirano, M. Nishikawa, T. Uehara, Curing reaction of epoxy resin composed of mixed base resin and curing agent: experimental and molecular simulations, Polymer 54 (2013) 46604668.

[25] http://accelrys.co.jp/products/materials-studio/

[26] Stewart. JJP. MOPAC (semiempirical quantum chemistry program based on Dewar and Thiel's NDDO approximation), http://openmopac.net/

[27] W. D. Cornell, P. Cieplak, C. I. Bayly, I. R. Gould, K. M. Merz, Jr., D. M. Ferguson, D. C. Spellmeyer, T. Fox, J. W. Caldwell, P. A. Kollman, A second generation force field for the simulation of proteins, nucleic acids, and organic molecules, J. Am. Chem. SOC. 117 (1995) 5179-5197. 
[28] D. Rosu, C. N. Cascaval, F. Mustata, C. Ciobanu, Cure kinetics of epoxy resins studied by non-isothermal DSC data, Thermochimica Acta 383 (2002) 119-27.

[29] D. Rosu, F. Mustata, C. Cascaval, Investigation of the curing reactions of some multifunctional epoxy resins using differential scanning calorimetry, Thermochimica Acta 370 (2001) 105-110.

[30] Direct Force Field 7.1 (Force field automation system), http:/www.aeontechnology.com.

[31] LAMMPS (Large-scale Atomic/Molecular Massively Parallel Simulator), Open source code, http://lammps.sandia.gov/. 
Molecular Dynamics Simulation of Crosslinked Epoxy Resins: Curing and Mechanical Properties

Tomonaga Okabe, Yutaka Oya, Koichi Tanabe, Gota Kikugawa and Kenichi Yoshioka 


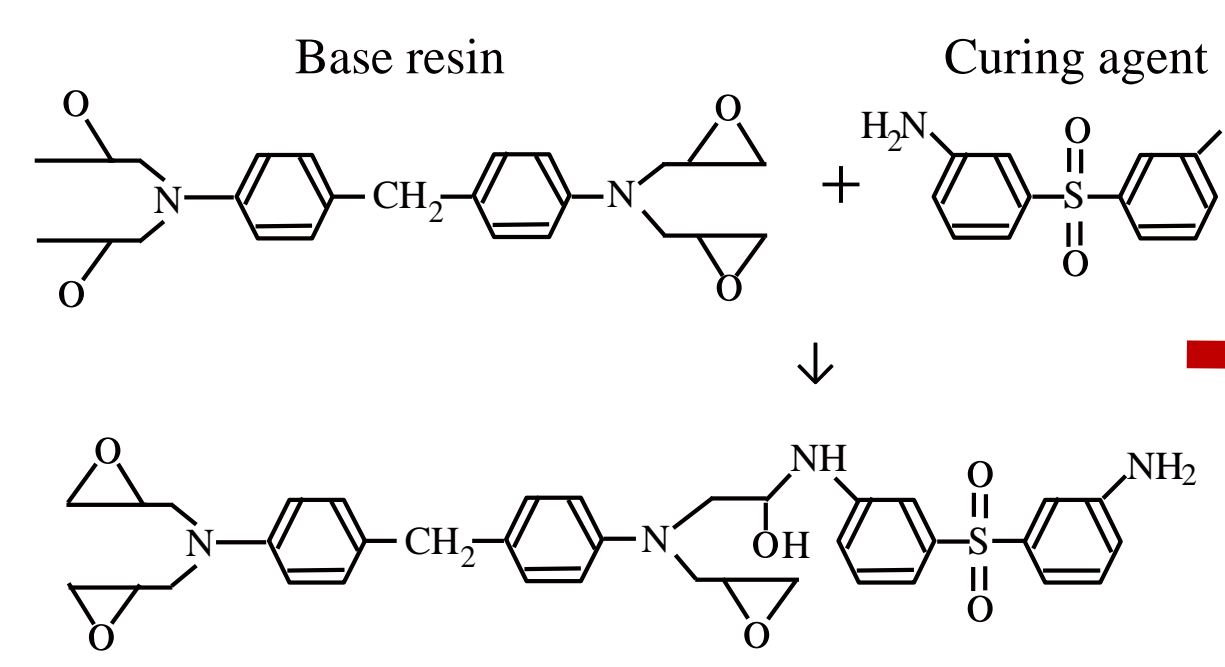

Curing reaction
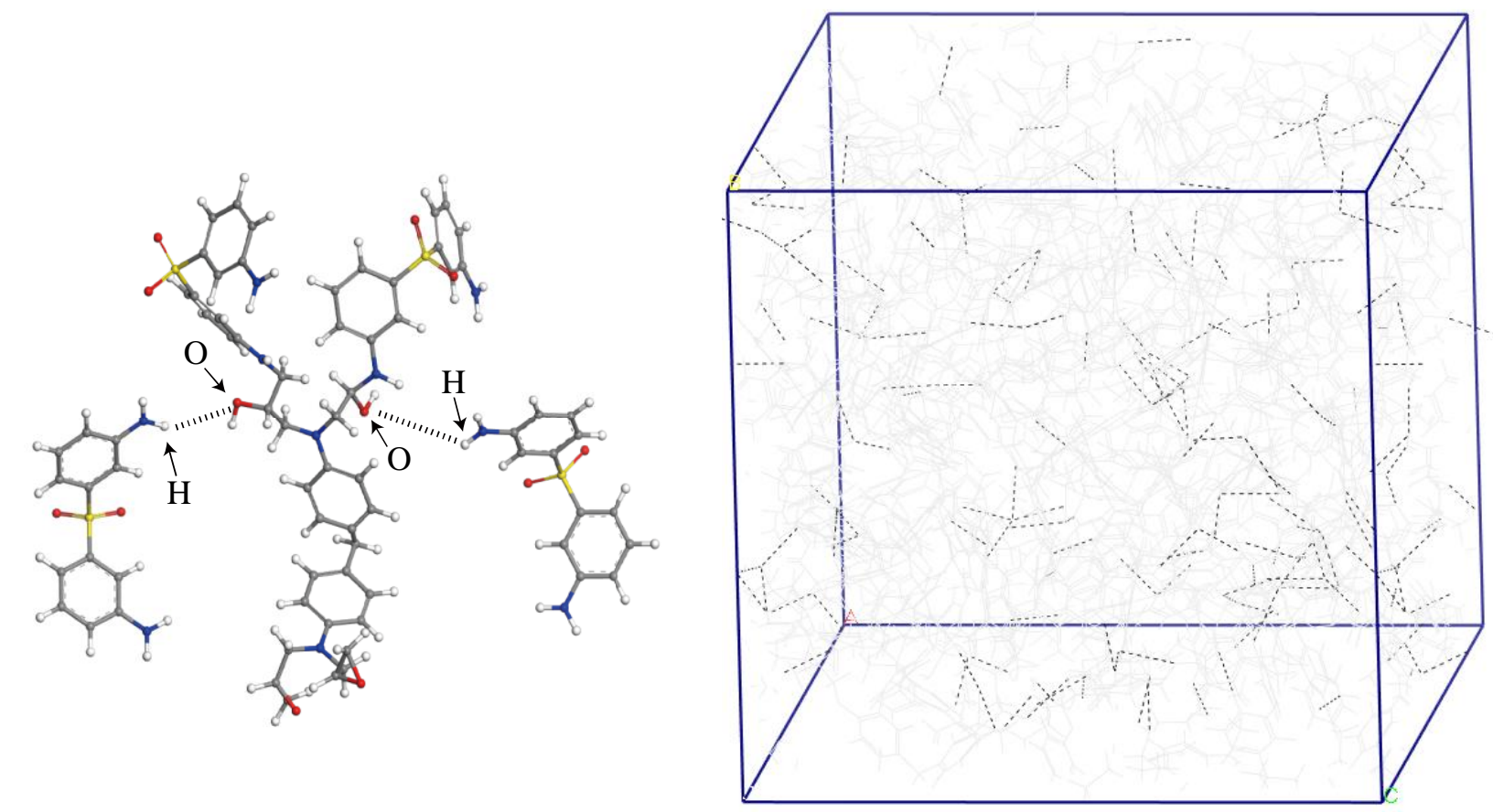

Network Structure by hydrogen bond interactions 